\title{
ZEUS high- $y$ Cross Section Measurement and Preparation for Low Energy Running
}

\author{
Shima Shimizu* \\ on behalf of the ZEUS collaboration \\ University of Tokyo \\ 7-3-1 Hongo, Bunkyo-ku, Tokyo 113-0033, JAPAN
}

\begin{abstract}
HERA is the only place to provide information on $F_{L}$ in the gluon dominated region of $e p$ interactions. The ZEUS experiment has extended its cross section measurement to the high- $y$ region, where the $F_{L}$ contribution can be sizable. In this report, details of the measurement and the first look on data from the Low Energy Running, which allows separation of $F_{2}$ and $F_{L}$, are presented.
\end{abstract}

\section{Introduction}

The $e p$ collider HERA has played a crucial role in the investigation of proton structure, especially in the low- $x$ region where gluons are dominant in the proton. The ZEUS experiment performed a precise measurement of Deep Inelastic Scattering (DIS) reduced cross sections using data collected in 1996 and 1997 [2]. The kinematics of lepton-proton DIS is described by $x$, the Bjorken scaling variable, $Q^{2}$, the negative square of the invariant mass of the virtual exchanged boson, and $y$, the inelasticy. The reduced cross sections can be defined as

$$
\tilde{\sigma} \equiv \frac{x Q^{4}}{2 \pi \alpha^{2} Y_{+}} \frac{d^{2} \sigma}{d x d Q^{2}}=F_{2}\left(x, Q^{2}\right)-\frac{y^{2}}{Y_{+}} F_{L}\left(x, Q^{2}\right),
$$

where $Y_{+}=1+(1-y)^{2}$. Since the contribution of $F_{L}$ to the cross section is sizable only at high $y$, the measurement provided information mainly on $F_{2}$, which is sensitive to the total number of quarks and anti-quarks in the proton. A measurement of $F_{2}$ also allows to determine a gluon distribution indirectly from the scaling violation, $\frac{\partial F_{2}}{\partial \ln Q^{2}}$.

The longitudinal structure function of the proton, $F_{L}$, is directly sensitive to gluon dynamics in the proton. Since high- $y$ corresponds to the low- $x$ region, $F_{L}$ with gluon dominance can be investigated at HERA. The ZEUS experiment has performed a new cross section measurement, which is optimized for the high- $y$ region, with data collected in 2006. A special trigger was prepared for this measurement, which takes events in a new kinematic region of high $y$. The measurement requires a good understanding of the scattered electron energy and a good control of background contamination, since events with higher $y$ have lower energy of the scattered electron ${ }^{\mathrm{a}}$, which is more difficult to identify. Details and results of the measurement are presented.

For separation of $F_{2}$ and $F_{L}$, cross sections at the same $\left(x, Q^{2}\right)$ but different $y$ values need to be measured (See Eq. 1). This requires measurements with different center-of-mass (CM) energies because of the relation $s=Q^{2} / x y$, where $s$ is the $e p$ center-of-mass energy. For that reason, HERA started the operation with lowered proton beam energy, called Low Energy Run (LER), in March 2007. Also shown in this report are the first LER data collected by the ZEUS experiment.

\footnotetext{
*JSPS (Japan Society for the Promotion of Science) research fellow, DC.

${ }^{a}$ The word electron is used for both electron and positron in this paper.
} 


\section{High- $y$ cross section measurement}

\subsection{Details of the analysis}

The data were collected with the CM energy of $318 \mathrm{GeV}$, where proton and positron beam energies were $920 \mathrm{GeV}$ and $27.5 \mathrm{GeV}$, respectively. The total luminosity was 29.5 $\mathrm{pb}^{-1}$.

For online event selection, two independent trigger-logic were prepared to take events with low scattered electron energy. One required a scattered electron with energy above $4 \mathrm{GeV}$. The second logic required $\Sigma_{\text {total }}\left(E-p_{z}\right)_{i}>30 \mathrm{GeV}$ and $\Sigma_{\theta_{i} \lesssim 165^{\circ}}(E-$ $\left.p_{z}\right)_{i}>20 \mathrm{GeV}$, where $E_{i}$ and $p_{z i}{ }^{\mathrm{b}}$ are energy and longitudinal momentum of the $i$ th cell of the calorimeter and each sum runs over all the cells and the cells excluding the ones in the rear, close to the beam pipe, respectively. Events satisfying at least one of the two triggers were selected.

For offline event selection, the following conditions were applied:

- $\left|\mathrm{Z}_{\mathrm{vtx}}\right|<50 \mathrm{~cm}$, where $\mathrm{Z}_{\mathrm{vtx}}$ is the $\mathrm{z}$ position of an event vertex.

- $\Sigma_{\text {total }}\left(E-p_{z}\right)_{i}>38 \mathrm{GeV}$

- The presence of an electron candidate found in the calorimeter with energy $\left(E_{e}>5 \mathrm{GeV}\right)$ and angle $\left(\theta_{e} \lesssim 170^{\circ}\right)$. For $\theta_{e} \lesssim 151^{\circ}$, a track was required to be matched to the candidate.

The electron energy scale and the dead material corrections are well understood based on the investigation of three data samples, namely exclusive $J / \Psi$ photoproduction $\left(E_{e} \lesssim 5\right.$ $\mathrm{GeV})$, QED Compton $\left(5 \mathrm{GeV}<E_{e}<25 \mathrm{GeV}\right)$ and DIS $\left(10 \mathrm{GeV}<E_{e}<35 \mathrm{GeV}\right)$. The uncertainty on the electron energy scale is assigned a value of $\pm 2 \%$.

The main source of contamination to the DIS sample are photoproduction $(\gamma p)$ events, $\gamma p \rightarrow X$, where the electron emits a quasi-real photon and disappears down the beam pipe. The photon interacts with the proton producing in particular $\pi^{0}$ and low energy $\pi^{ \pm}$, which may be misidentified as electron in the calorimeter. The contamination is severe at high- $y$, where the energy of the scattered electron is small. To understand the $\gamma p$ background, two analyses were performed. The first analysis was done with events tagged by the $6 \mathrm{~m}$ tagger, which is a detector placed downstream of the electron beam and which can directly detect an escaping electron. The sample is reasonably described by the $\gamma p$ MC except for the

${ }^{\mathrm{b}}$ In the ZEUS coordinate, proton beam direction is in the $z$ direction. 
overall normalization. The second analysis was done with a $\gamma p$ enriched sample, in which a low quality scattered electron was found, based on its calorimeter shower shape. In this sample, the difference between the data and the DIS MC is described by the scaled $\gamma p$ MC. The normalization factor for the $\gamma p \mathrm{MC}$ was extracted for each sample separately and the two values agreed within 5\%. Considering the imperfect description of the shape of the considered distributions by the $\gamma p \mathrm{MC}$, an uncertainty of $\pm 10 \%$ was conservatively assigned to the normalization factor.

Distributions of measured quantities for selected DIS events are shown in Fig. 1. They are well described by MC prediction in which the normalization factor described above is applied to the $\gamma p \mathrm{MC}$.

\subsection{Reduced cross sections}

Kinematic variables are reconstructed using the energy and the angle of the scattered electron. Bins are defined in the $\left(y, Q^{2}\right)$ plane for good coverage of the high- $y$ region. In the region where reduced cross sections were extracted, most of the bins had acceptance above $60 \%$ and the contamination of $\gamma p$ events was estimated to be less than $40 \%$ in each bin.

Reduced cross sections are extracted as,

$$
\tilde{\sigma}=\frac{N_{\text {data }}-N_{\gamma \mathrm{p} \mathrm{MC}}}{N_{\text {DIS MC }}} \cdot \tilde{\sigma}_{\text {theory }}^{\text {BOR }} .
$$

The following systematic sources were taken into account:

- electron energy scale $( \pm 2 \%)$,

- $\gamma p \mathrm{MC}$ normalization factor $( \pm 10 \%)$,

- electron finding inefficiency $( \pm 10 \%)$,

- $\Sigma_{\text {total }}\left(E-p_{z}\right)_{i}$ threshold $( \pm 2 \mathrm{GeV})$.

The effect on the cross section measurement was evaluated by varying the value of each systematic source by a factor in brackets. At highest $y$, for low $Q^{2}\left(Q^{2}<50 \mathrm{GeV}^{2}\right)$, the systematic uncertainty from the $\gamma p$ MC normalizaton factor was largest, giving $\sim 5 \%$ uncertainty on the cross section.

The measurement was done for $0.1<y \lesssim 0.8$ and $25 \mathrm{GeV}^{2}<Q^{2}<$ $1300 \mathrm{GeV}^{2}$. The extracted reduced cross sections are shown in Fig. 2, as a Figure 2: The reduced cross sections as a function function of $y$ for fixed $Q^{2}$ values. They of $y$ for fixed $Q^{2}$. are compared with the Standard Model predictions with CTEQ5d PDF [3] and with ZEUSJets PDF [4]. The measured reduced cross sections are well described by the predictions.

The measurement was successfully extended to the high- $y$ region. The measured points are extended to higher $y$ for $Q^{2} \lesssim 300 \mathrm{GeV}^{2}$, compared to the previous measurement, and more measured points are provided at mid- $y$ value $(y \gtrsim 0.3)$. 


\section{Low Energy Running (LER)}

HERA ended its operation with proton beam energy of $920 \mathrm{GeV}$ in March 2007. Since then, HERA started to operate with lowered proton beam energy, namely $460 \mathrm{GeV}$, for the DIS cross section measurement with different center of mass energies which will allow the extraction of $F_{2}$ and $F_{L}$.

The ZEUS experiment has performed a feasibility study for $F_{L}$ measurement [5]. As can be seen in Eq. 1, larger difference in $y$ between the measurements at different beam energies brings better precision for $F_{L}$. It can be achieved by a cross section measurement at higher $y$ in LER data. As presented in this report, the ZEUS experiment has already extended its measurement to higher $y$ compared to the previous measurement. The experiment started to collect LER data, as shown in Fig. 3, with a newly implemented trigger which requires $\Sigma_{\text {total }}\left(E-p_{z}\right)_{i}>30$

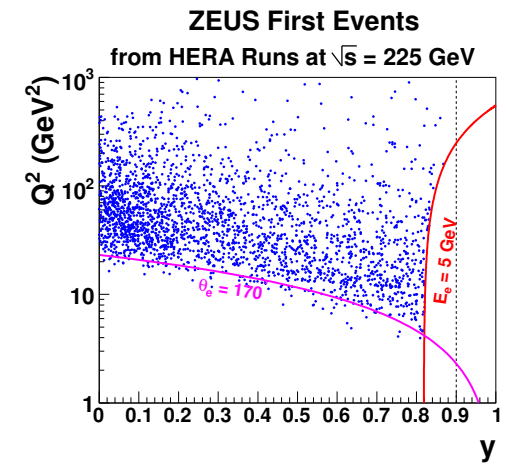

Figure 3: First look on the LER data, in the $\left(y, Q^{2}\right)$ plane.

\section{Summary}

The ZEUS experiment performed a new cross section measurement which is optimized for the high- $y$ region using data collected in 2006. The measurement extended to higher $y$ in the kinematic region, compared to the previous measurement. The experiment started to collect LER data, aiming for a direct measurement of $F_{L}$.

\section{References}

[1] Slides: http://indico. cern. ch/contributionDisplay. py? contribId=278\&sessionId=8\&conf Id=9499

[2] ZEUS Coll., S. Chekanov et al., Eur. Phys. J. C21 443 (2001).

[3] CTEQ Coll., H.L. Lai et al., Eur. Phys. J. C12 375 (2000).

[4] ZEUS Coll., S. Chekanov et al., Eur. Phys. J. C42 1 (2005).

[5] D. Kollar, Prospects for a measurement of $F_{L}$ at HERA with the ZEUS detector, in proceedings, DIS 2006 (2006) 